\title{
EDITORIAL
}

\section{It takes two to tango in the microenvironment!}

\author{
Josie Ursini-Siegel' and Morag Park*2 \\ See related research by Fordyce et al., http://breast-cancer-research.com/content/14/6/R155
}

\begin{abstract}
Using mortal non-tumorigenic human mammary epithelial cells and fibroblasts, Fordyce and colleagues show that an epithelial stress response promotes pro-tumorigenic changes in mammary fibroblasts. Fibroblast reprogramming was dependent on activin A or prostaglandin $E_{2}$ produced by epithelial cells and, in turn, promoted enhanced migration of epithelial cells. These events in epithelial cells in vitro, including telomere loss, heightened DNA damage response, and activin $A$ expression, are observed in breast ductal carcinoma in situ lesions surrounded by stroma bearing hallmarks of activated fibroblasts and immune and endothelial cell infiltration. Thus, reciprocal epithelial-stromal interactions facilitate progression to malignancy and occur even at the earliest stages of mammary tumorigenesis.
\end{abstract}

Extensive evidence supports an integral role for crosstalk between tumor cells and neighboring stromal cells, which promotes the growth of epithelial cancers and their progression toward increasing malignancy. The traditional view of these interactions posits that stromal components, including fibroblasts, endothelial cells, and a subset of immune cells, condition the microenvironment to favor tumor growth and metastasis by secreting growth factors, angiogenic factors, cytokines, and proteases. However, recent studies have convincingly demonstrated reciprocal crosstalk between the epithelial and stromal compartments, whereby tumor cells engage in paracrine signaling to increase the pro-tumorigenic properties of immune cells and fibroblasts within the stromal microenvironment. The article by Fordyce and colleagues [1] in a recent issue of Breast Cancer Research demonstrates convincingly that stromal-epithelial interactions, enhancing

*Correspondence: morag.park@mcgill.ca

${ }^{2}$ Goodman Cancer Research Centre, McGill University, 1110 Pine Avenue West, Montreal, QC, Canada, H3A 1 A3

Full list of author information is available at the end of the article the acquisition of malignancy, occur even at the initial stages of carcinogenesis.

Previous work by the Tlsty laboratory has identified activin $\mathrm{A}$, a member of the transforming growth factorbeta (TGF- $\beta$ ) family, as an integrator of tumor/stromal crosstalk [2]. They showed that, while human immortalized mammary epithelial cells (HMECs) undergo senescence in response to DNA damage or telomere erosion, HMECs lacking an intact $\mathrm{p} 16 / \mathrm{Rb}$ pathway are rendered hyper-proliferative in response to such genotoxic insults as a result of activin A-induced cyclooxygenase-2 (COX2) expression [2]. In their article in Breast Cancer Research, the authors demonstrate that epithelial-derived activin A is sufficient to increase the tumor-promoting properties of primary mammary fibroblasts in a COX2-dependent manner. These properties include increased depostion of extracellular matrix components and elevated production of growth factors and inflammatory cytokines and are highly reminiscent of a cancer-associated fibroblast (CAF) phenotype [1]. In contrast, a DNA damage stimulus applied directly to fibroblasts is sufficient to induce activin A-driven COX2 pro-inflammatory responses but cannot promote extracellular matrix deposition from primary mammary fibroblasts. Therefore, cell-extrinsic stress signals emanating from immortalized mammary epithelial cells are required to fully elicit a CAF-like phenotype in neighboring fibroblasts. These data suggest that immortalized mammary epithelial cells that have bypassed the $\mathrm{p} 16 / \mathrm{Rb}$ senescence checkpoint are exquisitely sensitive to DNA damage-induced oncogenic transformation as a consequence of both increased genomic instability and the acquisition of a pro-tumorigenic stromal microenvironment. It is likely that such epithelial stress-induced functions will extend to components of the immune lineage. Indeed, CAFs can promote a nuclear factor-kappa B (NF-kB)-driven pro-inflammatory response that facilitates the transition of hyperplastic lesions to overt carcinoma [3]. Moreover, activin A was recently shown to favor differentiation of macrophages with $\mathrm{M}_{1}$ type inflammatory properties, whereas inhibition of activin A signaling promotes $M_{2}$ macrophage polarization [4]. Indeed, macrophage infiltration into mammary tumors is required for the angiogenic switch and supports breast cancer metastasis [5]. 
Observations generated by the Tlsty laboratory suggest that the combined loss of tumor suppressors, such as p16INK4a, and stress signals induced from a DNA damage response are sufficient to convert primary epithelial cells into tumor promoters. In contrast, nontransformed mammary epithelial cells undergo senescence under these conditions [2]. When mixed with ErbB2-transformed mammary epithelial cells, normal mammary epithelial cells have the inherent ability to inhibit the tumorigenic phenotype whereby they secrete soluble factors that allow ErbB2-transformed mammary cells to reconstitute a normal, differentiated mammary gland instead of forming overt mammary tumors [6]. ErbB2-expressing cells isolated from these chimeric ductal structures retain their tumor-forming properties when injected into epithelium-free mammary fat pads [6], highlighting that signals from a normal mammary microenvironment, composed of stromal, epithelial, and host-mediated signals, may combine to suppress the cancer phenotype.

Fordyce and colleagues [1] demonstrate that activin A secreted from immortalized mammary epithelial cells following genotoxic stress is sufficient to induce a fibrotic response in neighboring fibroblasts. In a similar study, reactive oxygen species (ROS) released from cancer cells stimulated an oxidative stress response in adjacent fibroblasts and, in turn, increased their replicative potential by inducing a metabolic shift toward aerobic glycolysis [7]. Indeed, DNA damage induced by chemotherapeutic agents or telomere dysfunction is known to increase ROS production within tumor cells. This suggests that intracellular stress responses that occur within tumor cells, and perhaps immortalized epithelial cells, result in the elaboration of extracellular signals in the form of cytokines and free radicals, which activate a pro-tumorigenic stroma. In turn, oxidative stress within the CAFs can feed back onto the cancer cells to potentiate genomic instability, further increasing their malignant properties [8]. These observations have important clinical implications as residual ductal carcinoma in situ (DCIS) lesions that remain following neoadjuvant chemotherapy may paradoxically potentiate mammary tumorigenesis. Indeed, the presence of adjacent DCIS lesions in patients with human epidermal growth factor receptor 2-positive $\left(\mathrm{HER2}^{+}\right)$invasive ductal carcinoma (IDC) showed an inferior response rate to chemotherapy plus trastuzumab compared with patients who present with IDC only [9]. Hence, activin A produced following chemotherapyinduced DNA damage may increase the tumorigenicity of resident DCIS lesions or render remaining IDC tumors more refractory to treatment.

The study by Fordyce and colleagues [1] focused primarily on the contribution of chemotherapy or telomere dysfunction to trigger double-stranded breaks within mammary epithelial cells, leading to the observed fibrotic response in neighboring fibroblasts. Supporting this, quantitative measurements of telomere fusions, indicative of genomic instability, have established their presence at comparable levels in DCIS and IDC lesions but absence in normal breast [10]. It is likely that mammary epithelial cells will adopt other strategies to initiate stress-induced desmoplasia of mammary fibroblasts. For instance, human telomerase (hTERT) has been associated with additional roles beyond telomere maintenance in inhibiting intracellular stress signals. Indeed, a fraction of hTERT resides within the mitochondria, where it functions to directly limit ROS production in response to stimuli that promote oxidative stress by increasing the pool of available anti-oxidants [11]. Moreover, Fordyce and colleagues [1] demonstrate that hTERT overexpression in p16-deficient immortalized mammary epithelial cells suppresses the ability of mammary fibroblasts to deposit extracellular matrix and produce pro-inflammatory cytokines. Thus, elevated hTERT expression in HMECs may limit desmoplasia both by stabilizing telomeres and by inhibiting mitochondrial ROS production. Finally, the increased replicative capacity of DCIS lesions may itself contribute to the evolution of a protumorigenic stroma. For example, hyper-proliferation of cells within pre-neoplastic tissue results in reduced intracellular nucleotide pools, which in turn can lead to replication-induced DNA damage and increased genomic instability [12]. Therefore, the increased metabolic demands of DCIS lesions that have lost $\mathrm{G}_{1}$ or $\mathrm{G}_{2}$ cell cycle checkpoints (or both) may render them extremely adept at stress-induced conditioning of a tumorigenic stroma. In light of these observations, defining the integrity of cell cycle checkpoints within DCIS lesions may be an important diagnostic tool prior to initiating neoadjuvant chemotherapy.

\section{Abbreviations}

CAF, cancer-associated fibroblast; COX2, cyclooxygenase-2; DCIS, ductal carcinoma in situ; ErbB2, v-erb-b2 erythroblastic leukemia viral oncogene homolog 2; HMEC, human immortalized mammary epithelial cell; hTERT, human telomerase; IDC, invasive ductal carcinoma; Rb, retinoblastoma protein; $\mathrm{ROS}$, reactive oxygen species.

\section{Competing interests}

The authors declare that they have no competing interests.

\section{Acknowledgments}

Work in the authors' labs is supported by Canadian Institutes of Health Research (CIHR) operating grants (MOP-11545 and 106635) and a Terry Fox New Frontiers Team Grant to MP, who holds the Diane and Sal Guerrera Chair in Cancer Genetics at McGill University, and by grants from the Susan G Komen Foundation (KG110197) and CIHR (MOP-111143) to JU-S, who holds a CIHR new investigator salary award.

\section{Author details}

'Lady Davis Institute for Medical Research, 3755 Cote St. Catherine Road, Montreal, QC, Canada, H3T 1E2. ${ }^{2}$ Goodman Cancer Research Centre, McGill University, 1110 Pine Avenue West, Montreal, QC, Canada, H3A 1 A3. 
Published: 12 March 2013

\section{References}

1. Fordyce CA, Patten KT, Fessenden TB, Defilippis R, Hwang ES, Zhao J, TIsty TD: Cell-extrinsic consequences of epithelial stress: activation of protumorigenic tissue phenotypes. Breast Cancer Res 2012, 14:R155.

2. Fordyce C, Fessenden T, Pickering C, Jung J, Singla V, Berman H, TIsty T: DNA damage drives an activin a-dependent induction of cyclooxygenase- 2 in premalignant cells and lesions. Cancer Prev Res (Phila) 2010, 3:190-201.

3. Erez N, Truitt M, Olson P, Arron ST, Hanahan D: Cancer-associated fibroblasts are activated in incipient neoplasia to orchestrate tumor-promoting inflammation in an NF-kappaB-dependent manner. Cancer Cell 2010, 17:135-147.

4. Sierra-Filardi E, Puig-Kroger A, Blanco FJ, Nieto C, Bragado R, Palomero Ml, Bernabeu C, Vega MA, Corbi AL: Activin A skews macrophage polarization by promoting a proinflammatory phenotype and inhibiting the acquisition of anti-inflammatory macrophage markers. Blood 2011, 117:5092-5101.

5. Lin EY, Li JF, Gnatovskiy L, Deng Y, Zhu L, Grzesik DA, Qian H, Xue XN, Pollard JW: Macrophages regulate the angiogenic switch in a mouse model of breast cancer. Cancer Res 2006, 66:11238-11246.

6. Booth BW, Boulanger CA, Anderson LH, Smith GH: The normal mammary microenvironment suppresses the tumorigenic phenotype of mouse mammary tumor virus-neu-transformed mammary tumor cells. Oncogene 2011, 30:679-689.

7. Martinez-Outschoorn UE, Lin Z, Trimmer C, Flomenberg N, Wang C, Pavlides S, Pestell RG, Howell A, Sotgia F, Lisanti MP: Cancer cells metabolically "fertilize" the tumor microenvironment with hydrogen peroxide, driving the Warburg effect: implications for PET imaging of human tumors. Cell Cycle 2011, 10:2504-2520.
8. Martinez-Outschoorn UE, Balliet RM, Rivadeneira DB, Chiavarina B, Pavlides S, Wang C, Whitaker-Menezes D, Daumer KM, Lin Z, Witkiewicz AK, Flomenberg $N$, Howell A, Pestell RG, Knudsen ES, Sotgia F, Lisanti MP: Oxidative stress in cancer associated fibroblasts drives tumor-stroma co-evolution: a new paradigm for understanding tumor metabolism, the field effect and genomic instability in cancer cells. Cell Cycle 2010, 9:3256-3276.

9. von Minckwitz G, Darb-Esfahani S, Loibl S, Huober J, Tesch H, Solbach C, Holms F, Eidtmann H, Dietrich K, Just M, Clemens MR, Hanusch C, Schrader I, Henschen S, Hoffmann G, Tiemann K, Diebold K, Untch M, Denkert C: Responsiveness of adjacent ductal carcinoma in situ and changes in HER2 status after neoadjuvant chemotherapy/trastuzumab treatment in early breast cancer--results from the GeparQuattro study (GBG 40). Breast Cancer Res Treat 2012, 132:863-870

10. Tanaka H, Abe S, Huda N, Tu L, Beam MJ, Grimes B, Gilley D: Telomere fusions in early human breast carcinoma. Proc Natl Acad Sci U S A 2012, 109:14098-14103.

11. Indran IR, Hande MP, Pervaiz S: hTERT overexpression alleviates intracellular ROS production, improves mitochondrial function, and inhibits ROS-mediated apoptosis in cancer cells. Cancer Res 2011, 71:266-276.

12. Bester AC, Roniger M, Oren YS, Im MM, Sarni D, Chaoat M, Bensimon A, Zamir G, Shewach DS, Kerem B: Nucleotide deficiency promotes genomic instability in early stages of cancer development. Cell 2011, 145:435-446.

doi:10.1186/bcr3388

Cite this article as: Ursini-Siegel J, Park M: It takes two to tango in the microenvironment! Breast Cancer Research 2013, 15:102. 